\title{
How Often Do Physicians Address Other Medical Problems While Providing Prenatal Care?
}

\author{
Andrew Coco, MD, MS \\ The Lancaster General Research Institute, \\ and the Department of Family and Commu- \\ nity Medicine, Lancaster General Hospital, \\ Lancaster, Pennsylvania
}

\begin{abstract}
PURPOSE It is unknown to what extent physicians address multiple problems while providing prenatal care. The objective of this study was to determine the percentage of prenatal encounters with 1 or more secondary and tertiary nonobstetric diagnoses and compare rates between family physicians and obstetricians.
\end{abstract}

METHODS Using the National Ambulatory Medical Care Survey, 1995 -2004, I analyzed prenatal visits to family physicians' and obstetricians' offices. The outcome measure was the percentage of prenatal encounters with 1 or more secondary and tertiary nonobstetric diagnoses seen by family physicians and obstetricians.

RESULTS There were 6,203 visit records that met study criteria, representing 223 million visits to obstetricians and 21 million visits to family physicians. Of the prenatal encounters with a family physician, $17.6 \%$ (95\% confidence interval $[\mathrm{Cl}], 12.9 \%-22.4 \%)$ included 1 or more secondary and tertiary nonobstetric diagnoses compared with $7.8 \%(95 \% \mathrm{Cl}, 6.1 \%-9.6 \%)$ of prenatal encounters with an obstetrician $(P<.01)$. After controlling for other variables, being seen by a family physician, compared with being seen by an obstetrician, remained an independent predictor of a prenatal visit with an additional nonobstetric diagnosis $(\mathrm{OR}=2.57 ; 95 \% \mathrm{Cl}, 1.82-3.64)$.

CONCLUSIONS Family physicians diagnose nonobstetric problems frequently and considerably more often than obstetricians while providing prenatal care. This practice style enhances access to comprehensive primary care for women.

Ann Fam Med 2009;7:134-138. DOI: 10.1370/afm.915.

\section{INTRODUCTION}

mbulatory family medicine often requires practitioners to address
multiple problems during encounters. ${ }^{1-3}$ There is little information
about the extent to which other conditions are diagnosed when physicians are focused on providing maternity care. Showing that family physicians have a comprehensive approach to care during prenatal visits could have important implications for access and quality of health care for women. ${ }^{4}$

This study analyzed 10 years of the National Ambulatory Medical Survey (NAMCS), 1995-2004, to compare the extent to which family physicians and obstetricians concurrently diagnosed medical problems while providing prenatal care.

\section{METHODS}

The data for this study were derived from prenatal visits from the National Ambulatory Medical Care Survey (NAMCS) during the 10-year period from 1995 to 2004. A detailed description of the survey is avail- 
able from a related study and from the National Center for Health Statistics (NCHS), ${ }^{5,6}$ In addition to demographic factors, variables include self-selected physician specialty, whether the physician is considered a primary care physician (2003-2004 only), and up to 3 diagnoses coded according to the International Classification of Diseases (ICD-9-CM). ${ }^{7}$

We examined all office visits with the primary diagnoses of normal pregnancy, supervision of high-risk pregnancy, complications of pregnancy, or pregnancyassociated diagnoses (Figure 1). Three-digit ICD-9-CM codes for prenatal visits may include diagnoses related to labor, postpartum care, or some unspecified aspect

\section{Figure 1. Selection of prenatal visits, NAMCS 1995-2004.}

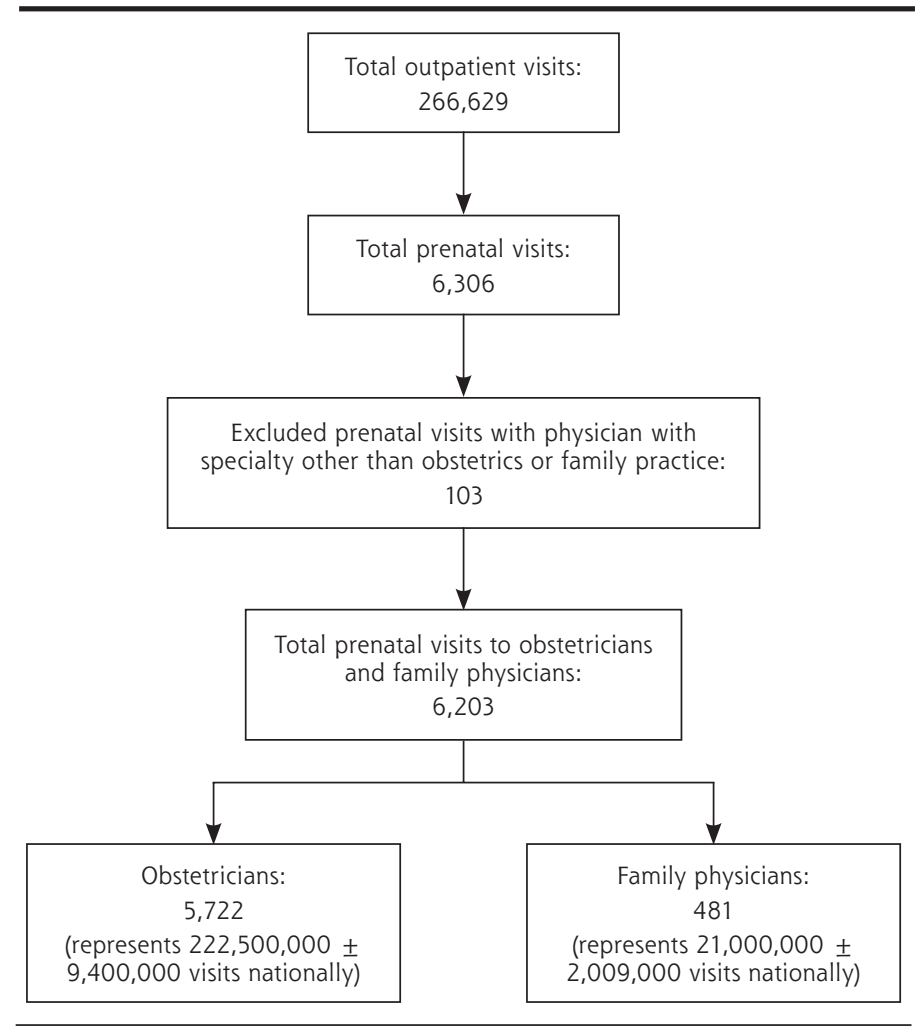

ICD-9-CM = International Classification of Diseases, 9th Revision, Clinical Modification; NAMCS = National Ambulatory Medical Survey.

Note: The following primary diagnoses were included as prenatal visits: normal pregnancy (ICD9-CM code V22), supervision of high-risk pregnancy (ICD-9-CM code V23), hemorrhage in early pregnancy (ICD-9-CM code 640), antepartum hemorrhage/abruptio placentae/placenta previa (ICD-9-CM code 641), hypertension complicating pregnancy/birth/puerperium (ICD-9-CM code 642), excessive vomiting in pregnancy (ICD-9-CM code 643), early or threatened labor (ICD-9CM code 644), prolonged pregnancy (ICD-9-CM code 645), other complications of pregnancy, not elsewhere classified (ICD-9-CM code 646), infection/parasite condition in mother classified elsewhere/complicated pregnancy/birth/puerperium (ICD-9-CM code 647), other current condition in mother classified elsewhere but complicating pregnancy/birth/puerperium (ICD-9-CM code 648), multiple gestation (ICD-9-CM code 651), malposition-unspecified (ICD-9-CM code 652), fetopelvic disproportion (ICD-9-CM code 653), abnormality of organs and soft tissues of pelvis (ICD-9-CM code 654), known or suspected fetal abnormality affect management of mother (ICD-9-CM code 655), other fetal and placental problems affecting management of mother (ICD-9-CM code 656), other problems associated with amniotic cavity and membranes (ICD-9-CM code 658), other indication for care/intervention related to labor/delivery not elsewhere classified (ICD-9-CM code 659), venous complications in pregnancy and the puerperium (ICD-9-CM code 671), and obstetric pulmonary embolism (ICD-9-CM code 673). of pregnancy care. Consequently, only visits in which the fifth digit of the 5-digit ICD-9-CM code was 3, he coded indicator for an antepartum condition or complication, were included. Additionally, visits to physicians whose specialty was not family medicine or bstetrics were excluded.

A variable was created indicating whether the secondary and tertiary diagnoses, if any, included a nonobstetric or medical condition. Visits with a secondary and, if listed, a tertiary diagnosis of an obstetric condition were excluded. The following diagnoses were excluded based on these criteria: primary diagnoses of normal pregnancy (ICD-9-CM code V22); supervision of high-risk pregnancy (ICD-9-CM code V23); antenatal screening (ICD-9-CM code V28); complications of pregnancy, childbirth, and the puerperium ((ICD-9CM codes 640-648, 651-656, 658-659, 671, and 673); and conditions originating in the perinatal period (ICD-9-CM codes 760-779). The rate of this variable - nonobstetric diagnosis - stratified by physician specialty represented the primary outcome of the study.

We calculated standard errors using Stata software, which accounts for the complex sampling design. Estimates based on less than 30 visit records are not considered accurate. ${ }^{6}$ Stata was programmed with the masked survey design variables using the svy package. ${ }^{8}$ Categorical variables were evaluated with the $\chi^{2}$ test. Stata, version 9 (StataCorp LP, College Station, Texas), was used to analyze all data. All $P$ values are 2tailed $P<.05$ was considered significant.

\section{RESULTS}

The prenatal visit selection process is outlined in Figure 1. After exclusions, 6,203 records, representing an estimated 222.5 million visits to obstetricians and $21.0 \mathrm{mil}$ lion visits to family physicians, remained in the study. The selection of visits with 1 or more secondary and tertiary non-pregnancy-related diagnoses is represented in Figure 2. Visits with both a secondary and a tertiary nonobstetric-related diagnosis were counted only once in this schema and for the statistical analysis. Table 1 displays a general overview of the frequency and types of nonpregnancy-related diagnoses stratified by physician specialty. The small number of visits per diagnostic cat- 


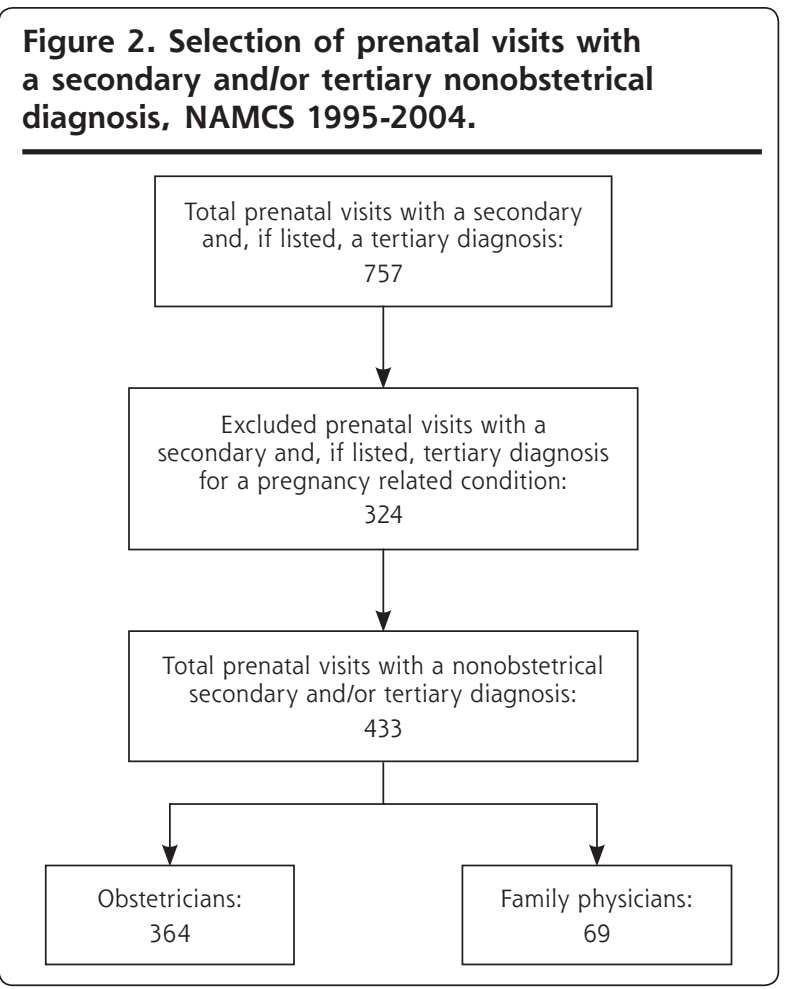

egory (less than 30) precluded statistical comparisons between specialties and calculation of meaningful percentage estimates. In contrast to the visit totals in Figure 2, Table 1 totals were higher because all secondary and tertiary diagnoses, if both present for the same visit, were counted separately.

The characteristics of the sample, grouped by physician specialty, are represented in Table 2 . Visits to family physicians tended to be by younger patients with higher rates of Medicaid and lower rates of private insurance. Family physicians were designated as the patient's primary care physician for $92 \%$ of their prenatal visits compared with $27 \%$ of obstetrician visits.

The primary outcome of the analysis is shown in the last row of Table 2. More than twice as many prenatal visits with family physicians $(17.6 \%$; $95 \%$ confidence interval $[\mathrm{CI}], 12.9 \%-22.4 \%)$, compared with obstetricians $(7.8 \% ; 95 \% \mathrm{CI}, 6.1 \%-9.6 \%)$, included 1 or more secondary and tertiary non-pregnancy-related diagnoses. Table 3 exhibits the results of the multivariate logistic regression model of whether a prenatal visit included an additional, non-pregnancy-related diagnosis. Independent predictors were a visit with a family physician (vs an obstetrician: adjusted odds ratio $[\mathrm{AOR}]=2.57 ; 95 \% \mathrm{CI}, 1.82-3.64)$ and nonwhite race (vs white race: $\mathrm{AOR}=1.36 ; 95 \% \mathrm{CI}, 1.03-1.81$ ) when adjusted for other demographic and insurance variables.
Table 1. Visit Counts by ICD-9-CM Diagnostic Categories for Prenatal Visits with 1 or More Secondary and Tertiary Non-Pregnancy-Related Diagnoses, NAMCS 1995-2004

Visits to obstetricians (ICD-9-CM diagnostic category)
Infectious and parasitic diseases (001-139)
Neoplasms (140-239)
Endocrine, nutritional and metabolic diseases, and
immunity disorders (240-279)
Diseases of the blood and blood-forming organs
(280-289)
Mental disorders (290-319)
Diseases of the nervous system and sense organs
(320-389)
Diseases of the circulatory system (390-459)
Diseases of the respiratory system (460-519)
Diseases of the digestive system (520-579)
Diseases of the genitourinary system (580-629)
Diseases of the skin and subcutaneous tissue (680-709)
Diseases of the musculoskeletal system and connective
tissue (710-739)
Congenital anomalies (740-759)
Symptoms, signs, and ill-defined conditions (780-799)
Injury and poisoning (800-999)
Supplementary classification of factors influencing health
status and contact with health services (V01-V85)
Total

Visits to family physicians (ICD-9-CM diagnostic category)

Infectious and parasitic diseases (001-139)

Neoplasms (140-239)

Endocrine, nutritional and metabolic diseases, and immunity disorders (240-279)

Diseases of the blood and blood-forming organs (280-289)

Mental disorders (290-319)

Diseases of the nervous system and sense organs (320-389)

Diseases of the circulatory system (390-459)

Diseases of the respiratory system (460-519)

Diseases of the digestive system (520-579)

Diseases of the genitourinary system (580-629)

Diseases of the skin and subcutaneous tissue (680-709)

Diseases of the musculoskeletal system and connective tissue (710-739)

Congenital anomalies (740-759)

Symptoms, signs, and ill-defined conditions (780-799)

Injury and poisoning (800-999)

Supplementary classification of factors influencing health 10 status and contact with health services (V01-V85)

Total

83

ICD-9-CM = International Classification of Diseases, 9th Revision, Clinical Modification; NAMCS = National Ambulatory Medical Care Survey.

\section{DISCUSSION}

This study analyzed 10 years of data from a nationally representative survey to characterize secondary and tertiary diagnoses associated with primary prenatal visits to family physicians and obstetricians. Family physicians diagnosed 1 or more secondary and ter- 


\begin{tabular}{|c|c|c|c|}
\hline \multirow[b]{2}{*}{ Characteristic } & \multicolumn{2}{|c|}{ Proportion of Visits, \% } & \multirow[b]{2}{*}{$P$ value } \\
\hline & $\begin{array}{l}\text { Family Physician } \\
(n=481)\end{array}$ & $\begin{array}{c}\text { Obstetrician } \\
(n=5,722)\end{array}$ & \\
\hline Age, years & 47.0 & 31.2 & $<.01$ \\
\hline$<24$ & 31.6 & 33.6 & $<.01$ \\
\hline $24-30$ & 21.4 & 35.2 & \\
\hline \multicolumn{4}{|l|}{$>30$} \\
\hline Race & 87.8 & 83.0 & .09 \\
\hline White & 7.0 & 11.8 & .09 \\
\hline Black/African American & 5.2 & 5.2 & .39 \\
\hline \multicolumn{3}{|l|}{ Latino } & \\
\hline Health insurance & 49.3 & 63.9 & $<.01$ \\
\hline Private & 36.5 & 21.0 & $<.01$ \\
\hline Medicaid & 4.7 & 2.7 & $<.01$ \\
\hline Self-pay & 9.5 & 12.4 & \\
\hline Other & 91.5 & 26.7 & \\
\hline Primary care physician ${ }^{a}$ & 15.5 & 12.9 & .21 \\
\hline High-risk diagnosis ${ }^{b}$ & 17.6 & 7.8 & $<.01$ \\
\hline Concurrent diagnosis & 17.6 & 7.8 & $<.01$ \\
\hline \multicolumn{4}{|c|}{ ICD-9-CM = International Classification of Diseases, 9th Revision, Clinical Modification. } \\
\hline \multicolumn{4}{|c|}{$\begin{array}{l}\text { a Data available only for } 2003 \text { and } 2004 \text {. } \\
\text { b Primary diagnosis of supervision of high-risk pregnancy (ICD-9-CM code V23) or complications } \\
\text { of pregnancy, childbirth, and the puerperium (ICD-9-CM codes 640-648,652-556, 658,658, 671, } \\
\text { and 673). }\end{array}$} \\
\hline
\end{tabular}

Table 3. Multivariate Logistic Regression Model Predicting Whether the Prenatal Visit Included 1 or More Secondary and Tertiary Nonobstetric Diagnoses

\begin{tabular}{lll}
\hline Predictor Variable & $\begin{array}{c}\text { Unadjusted } \\
\text { OR (95\% CI) }\end{array}$ & $\begin{array}{c}\text { Adjusted }^{\mathrm{a}} \\
\text { OR (95\% CI) }\end{array}$ \\
\hline Family physician (vs obstetrician) & $2.52(1.78-3.58)$ & $2.57(1.82-3.64)$ \\
Nonwhite race (vs white race) & $1.31(1.00-1.74)$ & $1.36(1.03-1.81)$ \\
\hline $\mathrm{Cl}=$ confidence interval; OR = odds ratio. & & \\
a Adjusted for age, insurance status, and Latino ethnicity. & \\
\hline
\end{tabular}

family medicine care is available. The results of this study provide an example of comprehensive care for women.

There were some aspects of the data that may limit the conclusions that can be drawn from our results. Diagnostic billing for a prenatal visit can be complex. Some insurance carriers bundle prenatal visits into the delivery code (billed at the time of delivery). When bundling occurs, obstetric billing is not necessary at the time of the visit, and medical diagnoses may be billed separately. When prenatal visits are unbundled, however, it becomes more problematic to bill separately for both prenatal care (based on gestational age and risk status) and another diagnosis at the time of the visit. Obstetricians, more so than family physicians, may not be accustomed to billing for other problems because a large percentage of their visits are prenatal with unbundled coverage. Several factors mitigate this concern. First, the diagnostic codes used in NAMCS are not derived from billing information. In NAMCS, physicians, or their staff, list diagnoses on a 1-page survey form near the time of the visit. These diagnoses are then coded by NAMCS staff at the NCHS central office. Survey participants are instructed that the intention is to capture the clinical scope of the encounter, not the sequence or number of problems that would lead to maximal reimbursement. Consequently, physicians of both specialties may be more likely to list secondary diagnoses on a survey form when not constrained by these financial implications. Second, it seems likely that

tiary non-pregnancy-related medical problems during $18 \%$ of their prenatal encounters compared with $8 \%$ for obstetricians. Family physicians served in the dual role of primary care physician and provider of prenatal care during $92 \%$ of maternity care patient encounters; the rate at which obstetricians provided primary care was considerably less, $27 \%$.

This practice style fits with the model for which family physicians are trained-to provide comprehensive primary care in the medical home ${ }^{9}$ — and these data show comprehensive primary care occurs even during prenatal visits. ${ }^{8}$ Family medicine is the only specialty in the United States providing comprehensive primary health care for women. Unlike men, women must go to a specialist to have routine and preventive health care for issues involving their reproductive organs unless family physicians routinely providing maternity care would also understand the constraints of secondary billing under an unbundled plan.

Aside from billing influences, there are several other data limitations. First, it was not possible to determine whether obstetricians recognized concurrent medical problems to the same extent as family physicians but, without coding for a diagnosis, referred patients to another physician for management. Such a practice style, if it were to occur, would still represent a less-comprehensive approach to care and not affect the study conclusions. Second, the analysis was limited by a small sample size within the diagnostic categories for the concurrent, non-pregnancy-related diagnoses. Although the overall rate of secondary and tertiary diagnoses was considerably higher in prenatal visits 
made to family physicians, conclusions about which diagnostic categories were differentially addressed between the two specialties could not be determined. Lastly, it was not possible to compare obstetric outcomes between specialties.

In conclusion, prenatal care by family physicians may benefit some women in terms of access to care by not having to schedule separate appointments for problems not related to prenatal care. A decline in prenatal care by family physicians could potentially affect access to nonobstetric services for pregnant women.

To read or post commentaries in response to this article, see it online at http://www.annfammed.org/cgi/content/full/7/2/134.

Key words: Prenatal care; comorbidity; concurrent diagnoses; family medicine

Submitted December 17, 2007; submitted revised June 30, 2008; accepted July 29, 2008.

A version of this article was presented at the American Academy of Family Physicians Scientific Assembly, September 17-21, 2007, Chicago, Illinois; and the Annual Meeting of the North American Primary Care Research Group, October 20-23, 2007, Vancouver, British Columbia.

Acknowledgments: Donna Cohen, MD, MS, provided critical review of the manuscript.

\section{References}

1. Stange $K C$, Zyzanski SJ, Jaén $C R$, et al. Illuminating the 'black box'. A description of 4454 patient visits to 138 family physicians. J Fam Pract. 1998;46(5):377-389.

2. Flocke SA, Frank SH, Wenger DA. Addressing multiple problems in the family practice office visit. J Fam Pract. 2001;50(3):211-216.

3. Beasley JW, Hankey TH, Erickson R, et al. How many problems do family physicians manage at each encounter? A WReN study. Ann Fam Med. 2004;2(5):405-410.

4. Brooks Barr W. Why pregnancy care should be an essential part of family medicine training. Fam Med. 2005;37(5):364-366.

5. Cohen DM, Coco AS. Declining trends in the provision of prenatal care visits by family physicians. Ann Fam Med. 2009;7(2)128-133.

6. NAMCS description. US Centers for Disease Control and Prevention Web site. http://www.cdc.gov/nchs/about/major/ahcd/namcsds.htm. Accessed Apr 3, 2007.

7. US Department of Health and Human Services, Centers for Disease Control and Prevention, Health Care Financing Administration. International Classification of Diseases, 9th Revision, Clinical Modification. 6th ed. Washington, DC: US Department of Health and Human Services; 2003.

8. Hing E, Gousen S, Shimizu I, Burt C. Guide to using masked design variables to estimate standard errors in public use files of the National Ambulatory Medical Care Survey and the National Hospital Ambulatory Medical Care Survey. Inquiry. 2003/2004;40(4):416-415.

9. Medical homes defined: principles establish basis for health system reform. AAFP News Now. March 6, 2007. 